\title{
Naloxone alteration of blood flow in a nonischemic region of the partially ischemic gerbil brain
}

\author{
DANLEY F. BROWN, PHD \\ STEPHEN P. LARSEN, PHD \\ CYRUS PARSA, DO
}

The effect of naloxone (NAL) treatment on cerebral blood flow in the partially ischemic gerbil brain was investigated. Under pentobarbital anesthesia, the right carotid artery in female gerbils was cannulated. A permanent ischemia was produced in the right anterior brain portion by carotid ligation. One hour after the surgical procedure, radioactive ${ }^{51} \mathrm{Cr}$, microspheres were injected via the carotid catheter before intraperitoneal administration of NAL (50 mg/kg) in saline solution. Five minutes later, radioactive ${ }^{46} \mathrm{Sc}$ microspheres were injected via the carotid catheter. Controls were injected in the same manner with vehicle and microspheres. Brain quarters were assessed for ${ }^{51} \mathrm{Cr}$ and ${ }^{46} \mathrm{Sc}$ radioactivity with a gamma counter. A significant decrease in microsphere deposition was observed with NAL treatment in the right rear, nonischemic part of the brain. No changes in radioactivity were detected in the ischemic portion. These findings suggest that NAL may alter brain blood flow independently of ischemia.

Administration of the opiate antagonist naloxone (NAL) facilitates various cardiovascular functions following endotoxic, hypovolemic, or spinal shock in experimental animals. ${ }^{1}$ Amelioration of spinal shock with NAL has been attributed ${ }^{2,3}$

primarily to maintenance of spinal cord blood flow in the injured area. Moreover, cortical somatosensory-evoked potentials were preserved in cats with spinal cord injury that were treated with NAL. ${ }^{3}$ These observations prompted investigators to determine whether NAL could alter brain blood flow following cerebral ischemia.

In 1982, Faden and associates ${ }^{4}$ demonstrated that NAL improved cortical somatosensory-evoked potentials in dogs following cerebral ischemia induced by carotid injection of air emboli. Hosobuchi and coworkers ${ }^{5,6}$ reported that NAL reversed neurologic deficits in gerbils with permanent unilateral carotid occlusion.

The mechanism of action of NAL presumably is at the level of the opiate receptor, and, therefore, NAL works by replacing the opiate agonist at the receptor site. The exact mechanism of action of reversed neurologic deficits remains unexplained. However, using a temporary carotid occlusion technique to produce cerebral ischemia in gerbils, Holaday and D'Amato ${ }^{7}$ and Kastin and colleagues ${ }^{8}$ found that the gerbil neurologic deficits were not altered with NAL treatment.

In addition, Gaines and coresearchers ${ }^{9}$ demonstrated that NAL administration had no beneficial effect on neurologic function or cerebral blood flow in conscious monkeys subjected to middle cerebral artery occlusion. Further, conflicting clinical reports $^{6,10,11}$ have been published on the efficacy of NAL in treating human cerebral ischemia.

To evaluate whether NAL treatment increases blood flow in ischemic brain tissue, we studied relative brain blood flow before and after NAL treatment in the partially ischemic gerbil brain. 


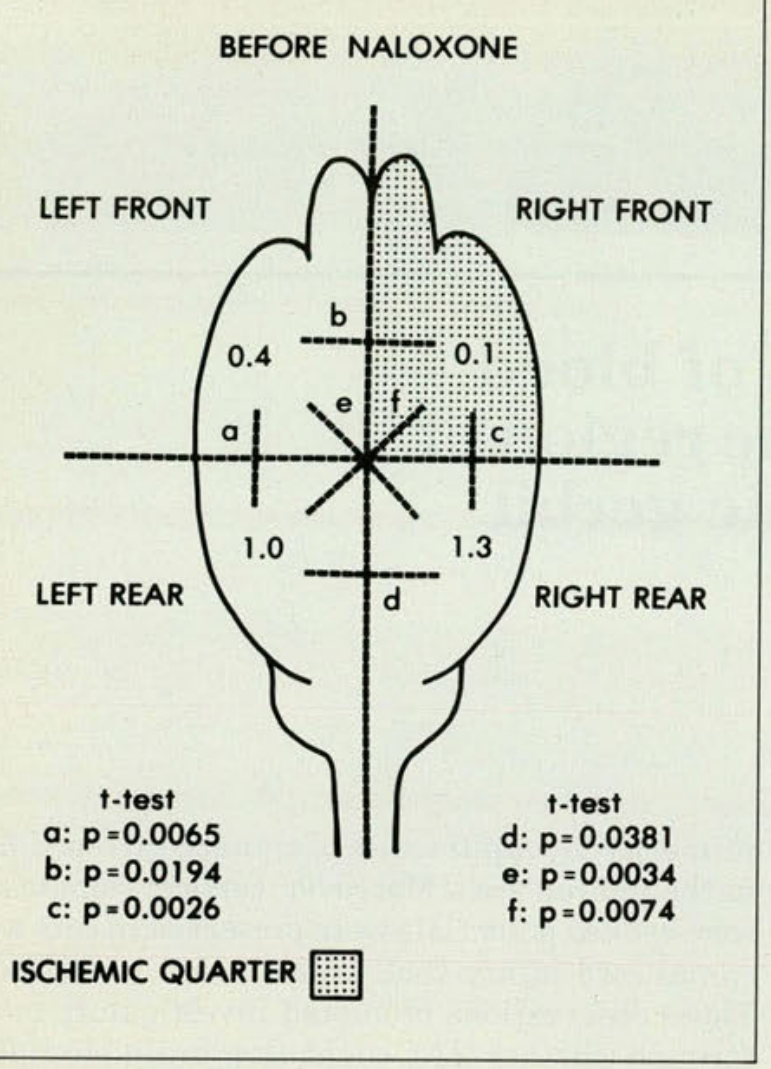

Fig 1. Radioactive distribution to the gerbil brain before naloxone administration.

\section{Materials and methods}

Female Mongolian gerbils weighing $50 \mathrm{~g}$ to $80 \mathrm{~g}$ were studied. The animals were housed at $23^{\circ} \mathrm{C}$ on a 12-hour light, 12-hour dark photoperiod, fed Purina Rat Chow, and given tap water ad libitum.

Twenty gerbils were anesthetized with pentobarbital $(50 \mathrm{mg} / \mathrm{kg})$ administered intraperitoneally. The right carotid artery was exposed and cannulated with a polyethylene (PE-10) catheter directed toward the aorta. The cathether was sutured in place and the carotid artery was ligated permanently. Because the gerbil usually has an incomplete circle of Willis (absence of a significant posterior communicating artery), the right front region of the brain was ischemic. ${ }^{12,13}$ Our data confirm this assumption.

Radioactively labeled (Chromium $\left[{ }^{51} \mathrm{Cr}\right]$ or Scandium [ $\left.{ }^{46 \mathrm{Sc}}\right]$ ) microspheres (New England Nuclear, Boston) $15 \pm 3 \gamma \mathrm{mm}$ in diameter were used for the blood flow measurements. ${ }^{14,15}$

One hour after surgery, a 0.1 -cc bolus of ${ }^{51} \mathrm{Cr}$ microspheres $\left(2.57 \times 10^{5}\right.$ beads, with a specific activity of $7 \mathrm{uCi}$ per $4.5 \times 10^{5}$ beads) was injected into the anesthetized gerbil via the carotid cathe-

\section{AFTER NALOXONE}

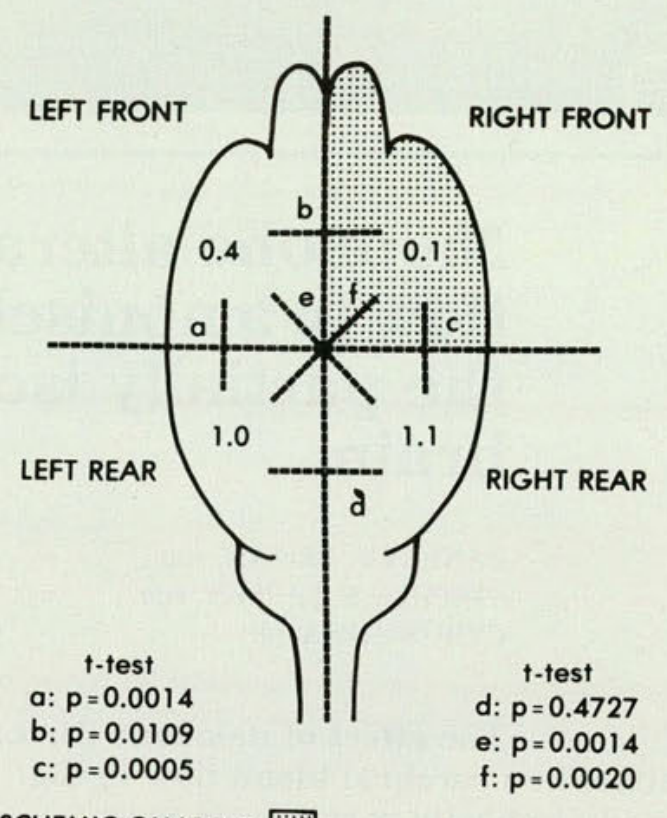

ISCHEMIC QUARTER

Fig 2. Radioactive distribution to the gerbil brain after naloxone administration.

ter into the central circulation. A saline flush $(0.05$ cc) ensured delivery of all of the microspheres. Five minutes later, NAL $(50 \mathrm{mg} / \mathrm{kg})$ in saline solution was given intraperitoneally in a volume equal to $1.0 \mathrm{~m} 1 / 100 \mathrm{~g}$ of body weight. The ${ }^{46} \mathrm{Sc}$ microspheres $\left(4.03 \times 10^{5}\right.$ beads, with a specific activity of 4.5 uCi per $4.5 \times 10^{5}$ beads) were administered five minutes after the NAL to determine blood flow after treatment.

Eight animals were injected intraperitoneally with saline vehicle and microspheres. Animals that did not receive both microsphere injections properly were eliminated from the statistical analyses. The figure legends note the numbers used in the data reduction process.

Five minutes after the second microsphere injection, the gerbils were sacrificed, and the brains were fixed for two days in Bouin's solution. After a brief period in $70 \%$ ethanol, the brains were dissected into quarters--left front and rear and right front and rear. Cuts were made along the anteroposterior midline and transversely at the level of the median eminence. Each brain quarter was weighed and analyzed for radioactivity with a 
Packard 5986 gamma counter. Corrections were made in the data for isotope energy overlap, counting efficiency, and sample geometry and weight.

Initially, t-tests were used to analyze for changes in radioactivity among brain quarters. Subsequent statistical differences in radioactive microsphere deposition before and after NAL or saline treatment were assessed by using a two-way analysis of variance, with repeated measures for time and location. Before this analysis, the data were transformed to a common base by using the ratio of counts in each brain region with respect to the left rear brain portion. Because the left rear portion is the least likely area to be affected by the right carotid artery ligation, this quarter was chosen as the reference area. A $P$ value of 0.05 or less was used to judge significance.

\section{Results}

The t-test analyses revealed significant differences in radioactivity among all brain areas before either saline solution or NAL administration (Figs 1,2). Following NAL treatment, a loss of significant difference $(P<0.05)$ between the left rear and right rear brain regions was noted compared with the controls (Fig 2, comparison d). Animals receiving saline vehicle alone displayed no changes in the relationship among brain quarters resulting from the saline injection.

Analysis of variance revealed a significant decrease $(P<0.021)$ in radioactivity in the right rear quarter of the brain (a nonischemic part of the brain) after NAL treatment (Fig 3). No other area, ischemic or nonischemic, demonstrated a significant alteration in microsphere deposition. Saline treatment had no significant effect on the distribution of radioactive microspheres in any brain region.

\section{Discussion}

The gerbil brain lacks the connection between the carotid and vertebrobasilar circulation, which makes the circle of Willis incomplete. ${ }^{12}$ Little anteroposterior blood flow exists in the gerbil brain; therefore, simple carotid ligation will prevent blood flow to an anterior portion of the brain. This vascular plan has made the gerbil brain an excellent model for studying cerebral ischemia. Our results confirmed this idea and demonstrated that the gerbils used in this study were ischemic centrally (Figs 1 and 2). Despite the fact that the carotid artery has no significant collateral branches, it is the main arterial source supplying the brain, and ischemia would be imperative following carotid ligation.

The questions of whether and how NAL treat- ment affords neurologic and brain blood flow recovery following ischemia still are debated. In this radiomicrosphere deposition study, we determined whether NAL had an effect on relative cerebral blood flow in the ischemic brain. Our data support a conclusion that NAL administered one hour after injury does not increase blood flow to brain areas that are ischemic from permanent right carotid ligation.

Faden and associates ${ }^{2}$ suggested that NAL treatment maintained cerebral blood flow in ischemic tissue at a level above the neuron-disabling range, although the average cerebral blood flow did not differ significantly from controls. The significant reduction in microsphere deposition we observed in the right rear brain quarter of NAL-treated gerbils can be supported by this idea. Our finding suggests that NAL altered the distribution of blood to brain regions not affected by the carotid occlusion of the partially ischemic gerbil brain, thus possibly producing a more equitable blood flow in the nonischemic area.

Virginia Gildengorin, $P h D$, assisted with the statistical analysis, and Gil Soo Kim, PhD, provided expert technical support.

The opinions or assertions in this article are the private views of the authors and are not to be construed as official or as reflecting the views of the Department of the Army or the Department of Defense. In conducting the research described in this report,

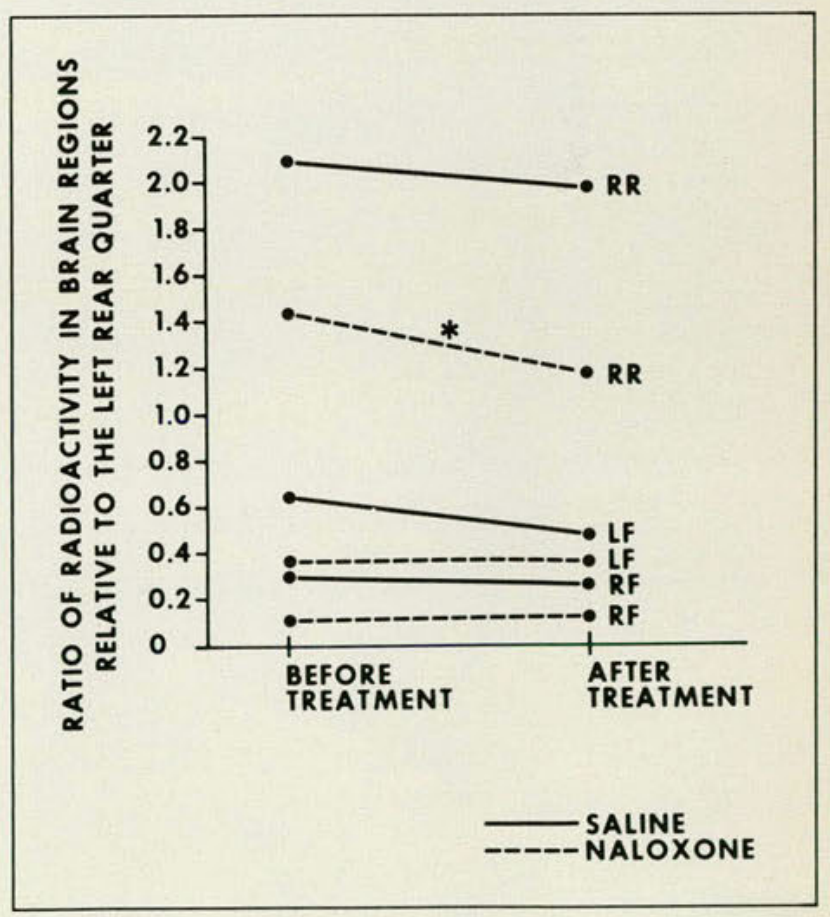

Fig 3. A plot of the ratios of the right front, right rear, and left front brain portion with respect to the rear region, before and after saline or naloxone treatment. 
the investigators adhered to the Guide for the Care and Use of Laboratory Animals as presented by the Committee on Revision of the Guide for Laboratory Animal Facilities and Care, Institute of Laboratory Animal Resources, National Research Council.

1. Holaday JW: Cardiovascular effects of endogenous opiate systems. Ann Rev Pharmacol Toxicol 1983:23:541-594.

2. Faden AI, Jacobs TP, Mougey E, et al: Endorphins in experimental spinal injury: Therapeutic effect of naloxone. Ann Neurol 1981;10:326332.

3. Young W, Flamm ES, Demonpoulos HB, et al: Effect of naloxone on post-traumatic ischemia in experimental spinal contusion. $J$ Neurosurg 1981;55:209-219.

4. Faden AI, Hellenbeck JM, Brown CQ: Treatment of experimental stroke: Comparison of naloxone and thyrotropin releasing hormone. Neurology 1982;32:1083-1087.

5. Hosobuchi Y, Baskin DS, Woo SK: Reversal of induced ischemic neurologic deficit in gerbils by the opiate antagonist naloxone. Science] 1982;215:69-71.

6. Hosobuchi Y, Baskin DS, Woo SK: Reversal of neurological deficits by the opiate antagonist naloxone after cerebral ischemia in animals and humans. J Cereb Blood Flow Metab 1982;2(suppl 1):S98-S100.

7. Holaday JW, D'Amato RJ: Naxolone or TRH fails to improve neurologic deficits in gerbil models of 'stroke.' Life Sci 1982;31:385-392.

8. Kastin AJ, Nissen C, Olsen RD: Failure of MIF-1 or naloxone to reverse ischemic-induced neurologic deficits in gerbils. Pharmacol Biochem Behav 1982;17:1083-1085.
9. Gaines C, Nehls DG, Suess DM, et al: Effect of naloxone on experimental stroke in awake monkeys. Neurosurgery 1984;14:308-314

10. Perraro F, Tosolini G, Pertoldi F, et al: Double-blind placebo-controlled trial of naloxone on motor deficits in acute cerebrovascular dis ease. Lancet 1984;1:915.

11. Baskin DS, Hosobuchi Y: Naloxone reversal of ischemic neurological deficits in man. Lancet 1981;2:272-275.

12. Vincent AL, Rodrick GE, Sodeman WA: The pathology of the Mon golian gerbil (Meriones unguiculatus): A review. Lab Anim Sci 1979;29:645-651.

13. Hamilton AJ, Black PM, Carr DB: Contrasting actions of naloxone in experimental spinal cord trauma and adrenal ischemia: A review. Neurosurgery 1985;17:845-849.

14. Levine BA, Sirinek KR, Gaskill HV III: The radiolabeled microsphere technique in gut blood flow measurement--current practice. J Surg Res 1984;37:241-255.

15. Heymann MA, Payne BD, Hoffman JI, et al: Blood flow measurements with radionuclide labeled particles. Prog Cardiovasc Dis 1977;20:55-79.

From the Department of Anatomy, University of Health Sciences College of Osteopathic Medicine, Kansas City (Drs Brown and Larsen); Division of Military Trauma Research, Presidio of San Francisco (Dr Brown); and Department of Pathology, UHSCOM (Dr Parsa).

Reprint requests to Dr. Brown, Department of Anatomy, University of Health Sciences College of Osteopathic Medicine, 2104 Independence Blvd, Kansas City, 64124. 